\title{
Enhanced Sensitivity Subwavelength Grating Waveguides for Silicon Photonics Sensing Applications
}

\author{
J. G. Wangüemert-Péreza , A. Hadij-ElHouati ${ }^{\mathrm{a}}$ A. Sánchez-Postigo a , J. Leuermann ${ }^{\mathrm{a}, \mathrm{b}}$, D. Pereira-Martín ${ }^{\mathrm{a}}$, \\ J.M. Luque-González ${ }^{a}$, P. Cheben ${ }^{\mathrm{c}}$, D.-X. Xu' ${ }^{\mathrm{c}}$, Jens H. Schmid ${ }^{\mathrm{c}}$, J. Čtyrokýd $^{\mathrm{d}}$, J. Soler-Penades ${ }^{\mathrm{e}}$, M. \\ Nedeljkovic $^{\mathrm{e}}$, G. Z. Mashanovich ${ }^{\mathrm{e}}$, A. Ortega-Moñux ${ }^{\mathrm{a}}$, R. Halir ${ }^{\mathrm{a}, \mathrm{b}}$, Í. Molina-Fernández ${ }^{\mathrm{a}, \mathrm{b}}$ \\ a Dpto. de Ingeniería de Comunicaciones, ETSI Telecomunicación, Universidad de Málaga, Campus de Teatinos s/n, 29071 Málaga, Spain \\ b Bionand Center for Nanomedicine and Biotechnology, Parque Tecnológico de Andalucía, 29590 Málaga, Spain \\ c National Research Council Canada, Ottawa, Ontario K1A OR6, Canada \\ d Institute of Photonics and Electronics, CAS, Prague, Czech Republic \\ e Optoelectronics Research Centre, University of Southampton, United Kingdom \\ Corresponding Author: gonzalo@ic.uma.es
}

\begin{abstract}
In this work we will review the enormous potential of subwavelength grating waveguides for sensing applications in the near and mid-infrared bands, demonstrating the capability to engineer the mode profile to maximize the light-matter interaction.

OCIS codes: $310.6628,130.6010,230.7370$
\end{abstract}

Silicon On Insulator (SOI) is the dominant platform for the implementation of photonic integrated devices in the near-infrared band (NIR). It is so advantageous for several reasons. Firstly, the high index contrast between silicon $\left(\mathrm{n}_{\mathrm{Si}}=3.476\right)$ and silicon dioxide $\left(\mathrm{n}_{\mathrm{SiO} 2}=1.444\right)$ allows us to obtain devices of compact size and waveguides with reduced bending radius (below $5 \mu \mathrm{m}$ ). Secondly, we can benefit from CMOS mature manufacturing processes, and thirdly, SOI exhibits low losses in the telecom optical wavelength band $(\lambda: 1.26-1.625 \mu \mathrm{m})$. However, the SOI platform also presents challenges. For example, the huge difference in mode dimensions makes it difficult to obtain high coupling efficiencies between the chip and the optical fiber, and the limited choice of refractive indexes offered by CMOS compatible materials makes it difficult to design devices with advanced characteristics. Furthermore, in the emerging and very promising mid-infrared band (MIR, $\lambda: 2-20 \mu \mathrm{m}$ ), where it is expected that a plethora of evanescent field absorption-based sensing applications will be developed in the near future, SOI use is constrained to the 2 to $4 \mu \mathrm{m}$ wavelength range due to high losses of the silicon dioxide for longer wavelengths.

The invention of subwavelength grating metamaterial waveguides (SWG), enabled by the continuous improvement of lithographic processes (minimum feature size below $100 \mathrm{~nm}$ ), has turned out to be pivotal for overcoming the aforementioned limitations. SWGs are periodic structures which are completely characterized by the following parameters: the pitch $(\Lambda)$ and the duty cycle (DC) or filling factor (see Fig. 1). To operate in the SWG regime, it is necessary to select the pitch small enough to avoid the diffraction. In doing so, the grating behaves as an equivalent homogeneous anisotropic medium. The potential of SWGs relies on the fact that, by using a unique etch-depth, it is possible to engineer the properties of the waveguide. By adequately choosing the duty cycle, the pitch, and, as demonstrated more recently, the tilt angle, is possible to respectively engineer the refractive index, its wavelength dependence and the birefringence of the equivalent metamaterial [1,2]. Making use of SWGs a broad range of novel and groundbreaking devices have been proposed mostly in the near infrared band. Mode converters, highly efficient fiber-to-chip couplers, ultra-broadband couplers, ultra-narrowband filters, polarization management structures, or mode de/multiplexers are

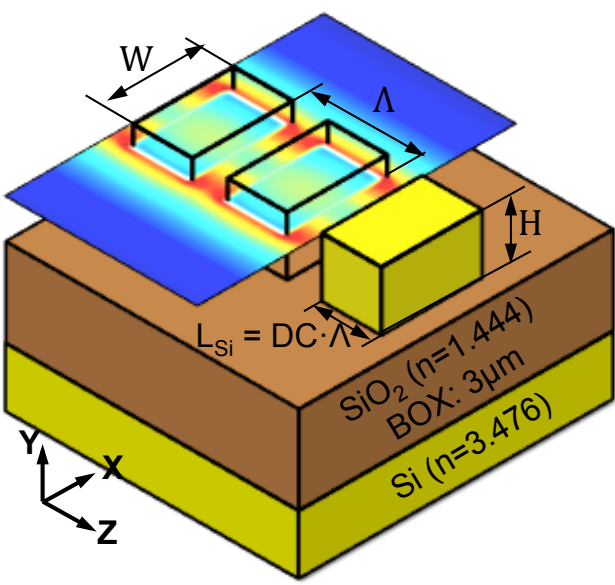

Fig. 1: Schematic representation of the SWG waveguide. Cut of the fundamental TE-Floquet-Bloch mode supported by the structure at half-height of the silicon blocks. only some examples of recently proposed devices [3].

SWG waveguides were first proposed for biosensing purposes in [4]. As can be seen in Fig. 1, the segmentation augments the light-matter interaction by simultaneously increasing the interaction volume (delocalizing the mode) and creating a high field intensity between the silicon blocks, wherein the analyte is also located. Since then, several 
research groups incorporated the SWG waveguides into their sensing architectures, mainly based on ring resonators based ones, experimentally validating their superior performance [5]. However, the majority of published SWG based works use the same dimensions as those used in standard silicon photonics wires (i.e. $W=450 \mathrm{~nm} ; \mathrm{H}=220 \mathrm{~nm}$ ), which might be sub-optimal from a sensitivity point of view. Recently, we have presented an in-depth study of sensing capabilities of SWG waveguides, in which the bulk and surface sensitivities have been calculated for a wide range of geometrical parameters [6]. Main conclusions of this study will be reviewed in this talk.

SWG waveguides have also become crucial to develop a low loss SOI based platform for the MIR band. The suspended silicon waveguide with SWG metamaterial cladding has shown to be a very attractive solution [7]. Based on removing the silicon buried dioxide (BOX) and anchoring the silicon core to the unsuspended lateral silicon areas by means of a subwavelength pattern of silicon bars, the resulting guiding structure is capable of covering the full transparency window of silicon, up to wavelengths of $8 \mu \mathrm{m}$ (losses below $3.1 \mathrm{~dB} / \mathrm{cm}$ have been reported at $\lambda=7.67$ $\mu \mathrm{m}[7])$. Most interestingly for sensing applications, by proper design, the suspended waveguides can exhibit a high light-matter interaction. The second goal of the talk will be to present our progress in the development of a complete set of basic building blocks for the silicon suspended platform (interconnecting waveguides, bends, grating couplers and MMIs, see Table 1). Besides, practical design rules to maximize the light-matter interaction but without excessively increasing the loss produced by the vertical and lateral leakage, will be also discussed.

Table 1. Performance summary of some basic building blocks of the suspended silicon waveguide platform

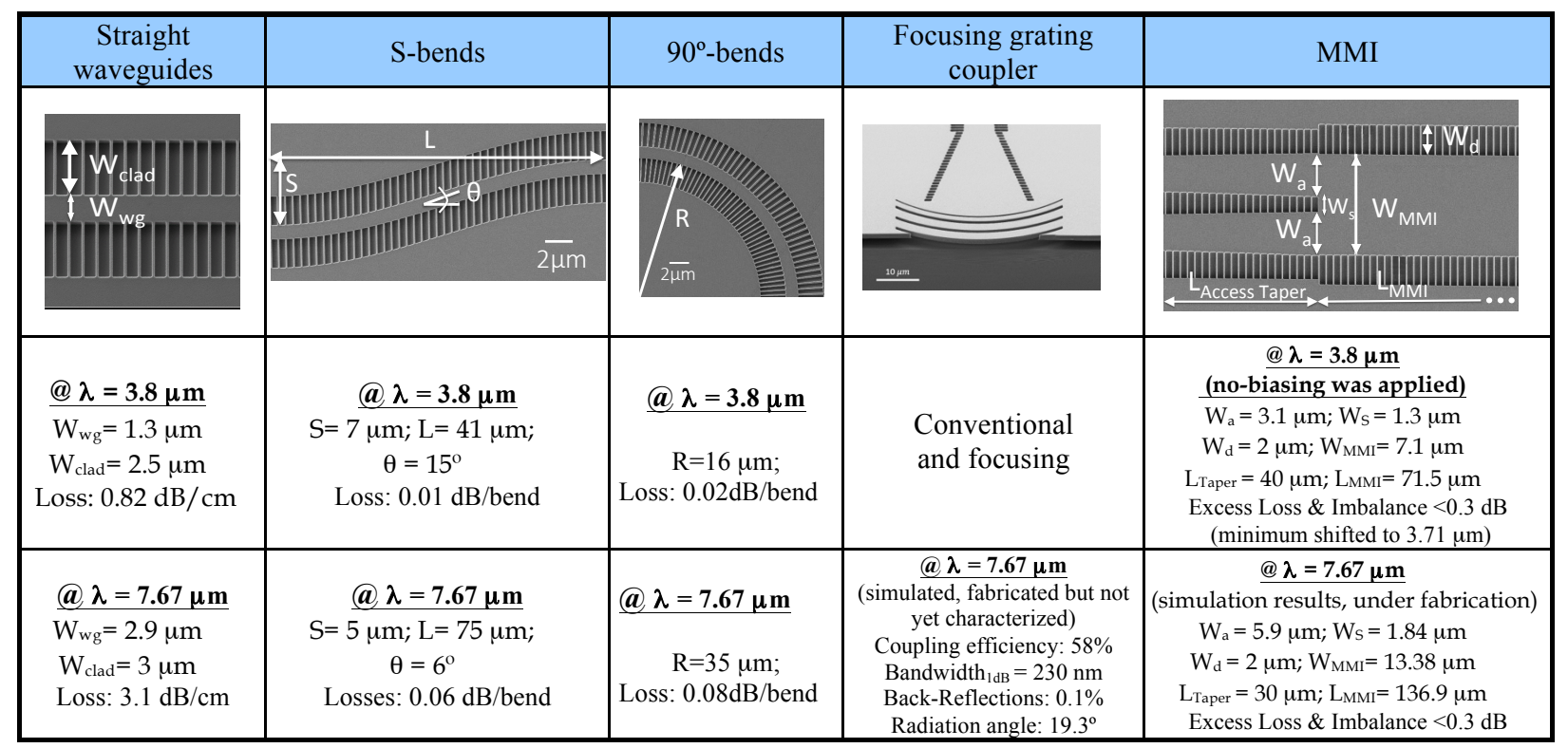

We acknowledge funding from: Ministerio de Economía y Competitividad (TEC2016-80718-R); European Union's Horizon 2020 research and innovation programme (Marie Sklodowska-Curie grant agreement No 713721); Ministerio de Educación, Cultura y Deporte (FPU14/06121, FPU16/03401, FPU16/06762, FPU17/00638); Universidad de Málaga (Andalucia TECH) and Czech Science Foundation (1900062S).

\section{References}

[1] P. Cheben et al., "Subwavelength integrated photonics", Nature, vol. 560, no 7720, p. 565, 2018.

[2] R. Halir et al., “Waveguide sub-wavelength structures: a review of principles and applications”, Laser Photon. Rev. 9 (1), pp. $25-49,2015$.

[3] R. Halir et al., "Subwavelength-Grating Metamaterial Structures for Silicon Photonic Devices", Proc. of the IEEE, no 99, pp. 1-14, 2018.

[4] J.G. Wangüemert-Pérez et al., "Evanescent field waveguide sensing with subwavelength grating structures in silicon-on- insulator", Opt. Lett.. 39, pp. 4442-4445, 2014.

[5] E. Luan, et al., "Silicon photonic biosensors using label-free detection", Sensors, vol. 18, no 10, p. $3519,2018$.

[6] J. G. Wangüemert-Pérez et al., "Subwavelength structures for silicon photonics biosensing”, Optics \& Laser Technology, vol. 109, p. 437$448,2019$.

[7] J. Soler Penadés et al., "Suspended silicon waveguides for long-wave infrared wavelengths", Optics letters, vol. 43, no 4, p. 795-798, 2018. 